\title{
Hybrid valence-bond states for universal quantum computation
}

\author{
Tzu-Chieh Wei \\ C. N. Yang Institute for Theoretical Physics and Department of Physics and Astronomy, \\ State University of New York at Stony Brook, Stony Brook, NY 11794-3840, USA \\ Poya Haghnegahdar and Robert Raussendorf \\ Department of Physics and Astronomy, University of British Columbia, \\ Vancouver, British Columbia, V6T 1Z1, Canada
}

(Dated: May 8, 2018)

\begin{abstract}
The spin-3/2 Affleck-Kennedy-Lieb-Tasaki (AKLT) valence-bond state on the hexagonal lattice was shown to be a universal resource state for measurement-based quantum computation (MBQC). Can AKLT states of higher spin magnitude support universal MBQC? We demonstrate that several hybrid 2D AKLT states involving mixture of spin-2 and other lower-spin entities, such as spin-3/2 and spin-1, are also universal for MBQC. This significantly expands universal resource states in the AKLT family. Even though frustration may be a hinderance to quantum computational universality, lattices can be modified to yield AKLT states that are universal. The family of AKLT states thus provides a versatile playground for quantum computation.

PACS numbers: 03.67.Ac, 03.67.Lx, 64.60.ah, 75.10.Jm
\end{abstract}

\section{INTRODUCTION}

Quantum computation by local measurements (MBQC) was shown to provide the same power of computation as the standard circuit model [1 5]. Studying various aspects of this model also offers new perspectives and insights, such as improvement of the resource overhead in linear-optics quantum computation 6, 7, utilization of topological protection [8, renormalization and holographic principles [7, 9, 10, exploration of quantum computational phases of matter [11 14 and the relation to symmetries [15] and contextuality [16]. There are still several aspects in MBQC to be understood, such as complete characterization of universal resource states and whether they can arise as unique ground states of gapped two-body interacting Hamiltonians $17-20$.

The cluster state on the square lattice was the first entangled state found to support universal MBQC [1]. Later its generalization to graph states on many $2 \mathrm{D}$ regular lattices were shown to be universal [21. Moreover, even faulty lattices can also support graph states that are universal for MBQC 22]. Given the success in graph states, one is led to ask the question: how to characterize all universal resource states? Specifically, do there exit other families of states like graph states that provide such versatility for quantum computational universality? Common properties shared by members in such families can provide insight to the relation between computational universality and physical properties, possibly providing stepping stones towards complete characterization of resource states, which is currently lacking.

In the following we shall investigate the family of the Affleck-Kennedy-Lieb-Tasaki (AKLT) states [23] and significantly expand known universal resource states in this family. Similar to graph states, AKLT states can be defined on any graph. In contrast to graph states composed uniformly of spin- $1 / 2$ entities, the local spin magntidue
$S$ of the AKLT states depend on the number $z$ of nearest neighboring sites, via $S=z / 2$; see e.g. Fig. 1. The entanglement stucture of the AKLT family is therefore different from that of the graph-state family. Graph states do not arise as unique ground states of two-body interacting Hamiltonians [17, which might be a disadvantage from the viewpoint of creating universal resource states by cooling. A finite gap in the Hamiltonian separating a unique resouce state as a ground state from excited states is thus a desirable feature [24]. With suitably chosen boundary conditions, AKLT states are unique ground states of certain two-body interacting Hamiltonians [23, some of which are believed to possess finite spectral gap [25, 26].

The insight why AKLT states might be useful for MBQC originated from the study of the 1D spin-1 AKLT state, shown to be capable of simulating one-qubit gates [3, 27. But the full quantum computational universality requires higher dimensions and was later established in the spin-3/2 AKLT state on the honeycomb lattice and a couple of other trivalent lattices 28 31. It is therefore natural to ask whether the university of the spin-3/2 AKLT state is fortuitous or there are other states, in particular ones with higher spins (than $3 / 2$ ), that can provide universal resource for MBQC? Furthremore, understanding why some states in the family are useful while others are not can provide connection of quantum computational universality to other physical properties.

Here we demonstrate that within the AKLT family many states defined on various $2 \mathrm{D}$ lattices contain mixture of different spin entities (such as $\operatorname{spin} S=1 / 2,1,3 / 2$ and 2) and provide universal resources for MBQC. (We note that spin- $1 / 2$ entities can occur at the boundary of the lattice.) The universality of this family is richer than the cluster family. Lattices possessing geometric frustration (i.e., loops with odd number of sites) may 
not support universal resource states but they can be modified (or decorated) so that the associated AKLT states are universal. The emerging picture from our study is the following. AKLT states involving spin-2 and other lower spin entities are universal if they reside on a two-dimensional (geometric) frustration-free lattice with any combination of spin-2, spin-3/2, spin-1 and spin$1 / 2$ (consistent with the lattice), provided that spin-2 sites are not neighbors. The constraint that the spin-2 states are not neighbors is due to the open question that whether AKLT states of uniform spin-2 entities can be universal, and it may be lifted if the anwser to that question is affirmative. We also note that, however, the existence of geometric frustration may not necessarily render the states non-universal, as we shall also demonstrate.

The task of proving universality of higher-spin AKLT states poses a greater challenge, as a straightforward extension of the generalized measurement or positiveoperator-valued-measure measurement (POVM) for the trivalent case does not work. Additional elements must be introduced to complete the spin-2 POVM [32], and as a result the known reduction of the AKLT states to graph states may no longer work. We shall provide a construction of spin-2 POVM (see Eqs. (2) below) that allows us to overcome this difficulty. We consider AKLT states on a few lattices, some of which are shown in Fig. 1, but the applicability of our method goes beyond these. For example, the lattice Fig. 1 1 a can be regarded as modified from the square lattice by replacing in the checker-board pattern spin- 2 sites with four spin- $3 / 2$ sites. On this lattice, spin- 2 sites do not reside as neighbors, but are surrounded by other spin-3/2 sites. Spin-3/2 sites can be dealt easily with a POVM composed of three directions (x, y and z); see Eq. (1) 28. Such modification makes the demonstration of the universality involving higher-spins possible via treating potential leakage errors on these sites. In a similar manner we shall also examine two other lattices (Figs. 1b and c) which support AKLT states with mixture of spin-2 and spin-1 entities. Moreover, we shall investigate the universality for the spin-2 AKLT state on the kagome lattice (Fig. 11) and the spinmixture AKLT states on the decorated kagome and star lattices (Fig. 17. and f), respectively. For convenience, we shall refer to these AKLT states defined on graphs with non-uniform vertex degrees as hybrid AKLT states or hybrid valence-bond states.

The structure of the remaining of this manuscript is as follows. In Sec. III we discuss an important in gredient of our proof for universality, i.e., the generalized measurement. It enables projection from four- or fivelevel states to effective two-level states, i.e., qubits. In Sec. III we describe a strategy to perform simulations without sampling the exact distribution of POVM outcomes. It is a worst-case scenario and therefore provides a 'lower bound' on universality. In Sec. IV we discuss consequences of geometric frustration for AKLT states on kagome and star lattices and provide some 'decoration' on these lattices that yied universal AKLT states. (a)

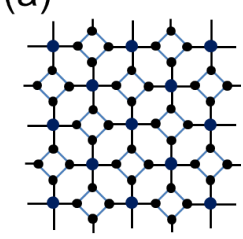

(d)

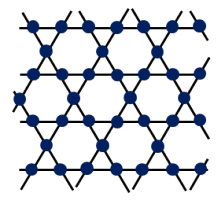

(b)

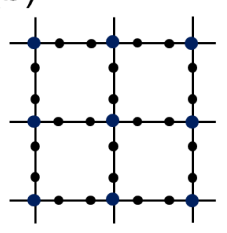

(e)

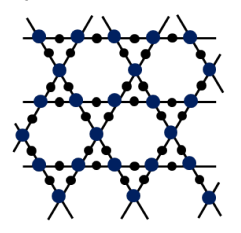

(c)

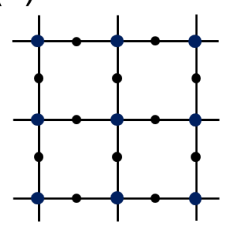

(f)

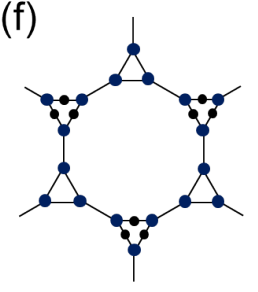

FIG. 1. (Color online) Lattices that host various AKLT states. By construction local spin magnitude $S$ depends on the coordination number $z$ by $S=z / 2$. (a) The lattice that supports a hybrid AKLT state with spin-3/2 and spin-2 mixture. It is obtained from the square lattice by replacing in the checker-board pattern spin-2 sites with four spin-3/2 sites. (b), (c) \& (e) Lattices that host spin-1 spin-2 hybrid AKLT states. (d) kagome lattice that hosts a spin-2 AKLT state. (f) A decorated star lattice which hosts a spin-3/2 spin-1 hybrid AKLT state.

We summarize in Sec. V

\section{THE GENERALIZED MEASUREMENT FOR REDUCTION TO QUBITS}

One approach for universality is the so-called quantum state reduction 33, i.e., to show that the state in question can be converted, via local measurements, to a known resource state, in particular some $2 \mathrm{D}$ graph state. The POVM for spin-3/2 sites consists of three rank-two elements 28.

$$
F_{\alpha}=\sqrt{\frac{2}{3}}\left(\left|S_{\alpha}=\frac{3}{2}\right\rangle\left\langle S_{\alpha}=\frac{3}{2}|+| S_{\alpha}=\frac{-3}{2}\right\rangle\left\langle S_{\alpha}=\frac{-3}{2}\right|\right),
$$

with $\alpha=x, y, z$. The POVM for the spin- 2 sites consists of three rank-two elements and three additional rank-one elements:

$$
\begin{aligned}
F_{\alpha} & =\sqrt{\frac{2}{3}}\left(\left|S_{\alpha}=2\right\rangle\left\langle S_{\alpha}=2|+| S_{\alpha}=-2\right\rangle\left\langle S_{\alpha}=-2\right|\right)(2 \mathrm{a}) \\
K_{\alpha} & =\sqrt{\frac{1}{3}}\left|\phi_{\alpha}^{-}\right\rangle\left\langle\phi_{\alpha}^{-}\right|,
\end{aligned}
$$

where $\alpha=x, y, z$ and $\left|\phi_{\alpha}^{ \pm}\right\rangle=\left(\left|S_{\alpha}=2\right\rangle \pm\left|S_{\alpha}=-2\right\rangle\right) / \sqrt{2}$. It can be verified that the completeness relation is satisfied: $\sum_{\alpha} F_{\alpha}^{\dagger} F_{\alpha}+\sum_{\alpha} K_{\alpha}^{\dagger} K_{\alpha}=\mathbb{1}$.

It has previously been shown [28, 30] that for the state $|\psi\rangle \sim \otimes_{v \in V} F_{\alpha_{v}, v}\left|\psi_{\mathrm{AKLT}}\right\rangle$ (where $v$ is a site index) is an encoded graph state on domains, regardless of $\alpha_{v}$ 's (the 


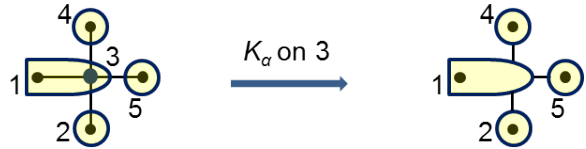

FIG. 2. (Color online) Code reduction. Sites $1 \& 3$ are in the same domain, and 2,4,5 are each a distinct domain. A POVM $K_{\alpha}$ on the spin- 2 site 3 is equivalent to removing site 3 without affecting the graph. Shapes such as circles (singlesite) and half-ovals (multi-site) are domains. On the l.h.s. an internal edge (between sites 1 and 3) is shown.

proof applies to any spin $S$ ). By a domain we mean a collection of connected sites on the original lattice that have outcomes associated with the $F$ 's with the same labeling $\alpha$. A domain is effectively a qubit. Outcomes associated with $K_{\alpha}$ are thus undesired, and we might need to regard them as "errors" 34]. Nevertheless, the $K$ operators can be rewritten as $K_{\alpha}=\sqrt{1 / 2}\left|\phi_{\alpha}^{-}\right\rangle\left\langle\phi_{\alpha}^{-}\right| F_{\alpha}$. This suggests that we can regard the outcome associated with $K_{\alpha}$ as arising from a two-step process: (1) first a result in the outcome associated with $F_{\alpha}$ is obtained; (2) then a further measurement is done in the basis $\left|\phi_{\alpha}^{ \pm}\right\rangle$ and the result $\left|\phi_{\alpha}^{-}\right\rangle$is obtained. A measurement in the basis $\left|\phi_{\alpha}^{ \pm}\right\rangle$corresponds to a measurement in the effective logical $X \equiv \sigma_{x}$ or $Y \equiv \sigma_{y}$ basis [35, respectively.

By construction the $K_{\alpha}$ 's outcomes only occur on spin2 sites. For each spin-2 site that is contained in a multisite domain, the effect of the measurement in the basis $\left|\phi_{\alpha}^{ \pm}\right\rangle$correspond to code reduction, namely, shrinking the number of sites inside the domain by one without affecting the quantum correlations of the present domain with others (i.e., the graph remains the same) [28; see e.g. Fig. 2. Therefore, it has no effect on the graph for the encoded graph state. However, for a single-site spin-2 domain, the measurement outcome $K_{\alpha}$ amounts to either a logical $X$ or $Y$ measurement on this logical qubit of the graph state. For the purpose of discussion we refer to them as X or Y undesired measurement, respectively. Pauli measurements on a graph state simply results in another graph state, whose graph can be easily deduced from simple rules [36. However, these Pauli measurements do not preserve planarity of graphs. In order to apply the percolation argument for planar graphs [28, 30, we need to somehow actively perform further measurement to recover planarity of the graphs, as will be discussed below.

The above discussions suggest that one approach for establishing universality is to employ certain procedure to recover the planarity of the graphs, caused by the $K_{\alpha}$ outcomes on single-site spin- 2 domains. Consider the undesired Y measurement. Its effect is to induce local complementation on the graph [36]. When the spin-2 domain is connected to three or few other domains, the planarity is preserved. When it is connected to four other domains (four is the maximum due to the geometry considered here), we can simply apply an additional Pauli Z mea- (a)

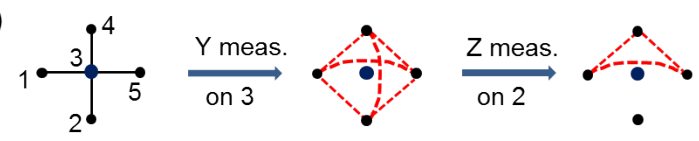

(b)

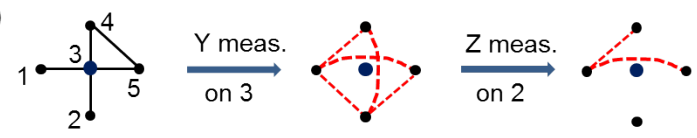

(c)

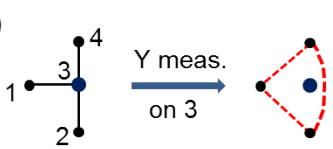

(d)

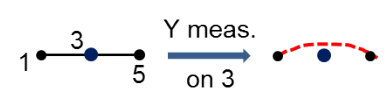

(e)

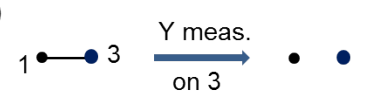

FIG. 3. (Color online) Graph transformation rules by $\mathrm{Y}$ measurement on a single-site spin-2 domain (site 3). For simplicity we assume every site is a domain. (a) and (b) illustrate the case where four distinct domains are connected to center spin-2 site. In (b) there is an additional edge between domains 4 and 5. To further make the graph remain planar, a Pauli Z measurement is made on any of the neighboring domain, say, 2. (c) shows the case where three distinct domains are connected to the spin-2 site. (This case can arise, e.g., as one of the neighboring domains was deleted in the second step of (a) or (b) associated with other spin-2 site.) (d) and (e) exemplify the cases of, respectively, two and one domain connected to a spin-2 site. Note that the above list does not exhaust all possibilities but just serves to illustrate that the undesired Y measurement can be treated to maintain planarity.

surement on any neighboring domain so as to recover planarity. See Fig. 3 for illustration of these measurements. The undesired X measurements are more troublesome, however, as they can result in longer-range connectivity (edges), and the planarity is more complicated to restore (it also depends on which reference neighbor to use for applying the graph rule [36]). We shall take the simplest approach by measuring logical $\mathrm{Z}$ on all their neighbors, thereby removing both the domains with undesired $\mathrm{X}$ measurement and their neighboring domains. This can be costly, but simplifies the simulations. In summary, we shall first treat all the undesired $\mathrm{X}$ measurement and then Y measurement to recover the full planarity.

\section{WORST-CASE SCENARIO}

We have just described a procedure to deal with $K_{\alpha}$ outcomes. However, in order to perform simulations to determine the computational universality, we need a method to sample $F$ 's and $K$ 's from the exact distribution according to the correlation in the AKLT states. Via exact sampling, we can also determine the probability of success for reducing the AKLT to a universal graph state. The exact sampling amounts to knowing the probability distribution of measuring Pauli $\mathrm{X} / \mathrm{Y}$ on some subset of 
qubits in a graph state. Although it is a linear-algebra problem in principle, obtaining the solutions and performing the sampling may be nontrivial. Fortunately, it turns out that we can avoid the exact sampling by considering the 'worst scenario' and still demonstrate universality (with unit probability) for various AKLT states.

Using the 2-step viewpoint of $K$ 's, we first sample POVM outcomes according to $F$ 's, which was solved in Ref. 28. The exact sampling could be obtained by assigning some spin-2 sites to flip from $F_{\alpha}$ to $K_{\alpha}$ according the exact probability distribution had one solved the linear-algebra problem mentioned above [37. Instead of sampling the exact distribution we then use what we call 'the worst-case scenario': all the spin-2 sites with $F_{\alpha}$ are flipped to $K_{\alpha}$. Then we implement the procedure in the previous section to recover full planarity. The resulting graphs are checked to see if they reside in the supercritical phase of percolation or not. Certifying that they are deep in the phase can be done by performing percolation 'experiment' (such as deleting vertices or edges) on these graphs [28]. If the graphs (before deletion) are in the supercritical phase, as the probability of deletion increases, there will be a clear signature of phase transition, beyond which no spanning cluster exists.

In order for universal quantum computation on graph states, the graph needs to possess a macroscopic number of traversing paths. This means that that the random graphs generated by our procedure need to reside inside the supercritical phase. Sitting right at the percolation transition is not sufficient for universal MBQC. We have checked the for the size $L$ large enough, $p_{\text {span }}$ is unity for random graphs resulting from the procedure on the AKLT states in Fig. 1 1a-c. But checking just this is not sufficient for establishing universality, as this does not rule out that the possibility that the graphs are sitting at the transition point nor that the graphs may be close to one-dimensional-like structure or star graphs (both being non-universal for MBQC). We have further performed site percolation simulation on the resultant graphs, shown in Fig. 4 a-c. It is seen that the graphs after the above procedure reside in the supercritical phase (with unit probability), even in the worst-case scenario. The significance of performing $p_{\text {span }}$ vs. $p_{\text {delete }}$ simulations is therefore to first prove an existence of phase transition, justifying the separation of two distinct phases and therefore the existence of macroscopic numbers of traversing paths (in particular at $p_{\text {delete }}=0$ ) in the supercritical phase of percolation. This shows that the hybrid AKLT states on Fig. 1a-c are universal resources for MBQC.

\section{GEOMETRIC FRUSTRATION: KAGOME AND STAR LATTICES}

For antiferromagnets, geometric frustration often gives rise to unexpected features. It was previously found in the star lattice that geometric frustration may inhibit the
AKLT state from being universal [31. Here we argue that it is the case in the kagome lattice, but show that for both lattices, we can decorate them so as to obtain universal AKLT states.

\section{A. The spin-2 AKLT state on the kagome lattice}

For the spin-2 only AKLT states, the worst-case scenario cannot be directly applied, as all the sites in each domain have some probability to be flipped from $F_{\alpha}$ to $K_{\alpha}$. However, even if we assume the best-case scenario, i.e., without flipping $F_{\alpha}$ 's to $K_{\alpha}$ 's, the graphs from sampling only the $F$ 's outcomes do not possess traversing paths for large enough lattice size. Thus, the spin-2 AKLT state on the kagome is not likely universal. We remark that what we have not ruled out is the possibility of other POVMs that might have enabled universal quantum computation.

The main reason for the lack of universality is due to the antiferromagnetic property in the AKLT state and the geometric frustration in the lattice. Due to the frustration in a triangle, the POVM outcomes with $(x, x, x)$, $(y, y, y)$ and $(z, z, z)$ cannot appear, similar to the star lattice 31]. This gives rise to an average probability of $p_{\text {delete }}^{\text {[bond] }}=1 / 2$ to remove an edge from an triangle, or equivalently the probability of occupying an edge being $1-p_{\text {delete }}^{\text {[bond] }}=1 / 2$, lower than the bond percolation threshold for the kagome lattice $p_{\mathrm{th}}^{[\text {bond }]} \approx 0.5244$. Therefore, the random graphs are not in the supercritical phase, implying non-universality for the original AKLT state. This intuitive argument does not take into account correlated errors and hence correlated edge deletion, but that is exactly what our simulations have dealt with.

\section{B. Decorated Kagome lattice: spin-1 spin-2 mixture}

To put to test the emerged picture that the AKLT state on any frustration-free lattice of at most degree- 4 is universal, we decorate the Kagome lattice by putting on each edge a vertex, removing frustration. This decorated lattice then hosts a spin-1 spin- 2 mixture hybrid AKLT state. For the lattice with $L \times L$ unit cells (each having 3 spin-2 particles and 6 spin-1 particles per cell) there are a total number $N=9 L^{2}$ of spins. Our simulations show that for $L$ large enough (e.g. $L \gtrsim 140$ ) our procedure yields random graphs in the supercritical phase with almost unit probability. The results of site percolation simulations are shown in Fig. 5 a. Even though the threshold of $p_{\text {delete }}$ is small, around 0.03 , it is still nonzero and therefore the random graphs are residing inside the supercritical phase. In summary, by decorating a frustrated lattice we are able to make the resultant AKLT state universal. 


\section{Decorated star lattice}

Whether the existence of geometric frustration will completely destroy the universality may depend on the particular lattice. It was shown that the spin-3/2 AKLT state on the star lattice is not likely universal [31. But we can decorate the star lattice by placing an additional vertex (which corresponds to a spin $S=1$ site) on each edge of all upside-down triangles; see Fig. 1f. There is still geometric frustration due to the remaining triangles and one might expect the associated AKLT state might not be universal. However, our simulations show that the resulting graphs after POVM are with probability one in the supercritical phase. The results of site percolation simulations are shown in Fig. 5 a. Therefore, the associated spin-3/2 spin-1 hybrid AKLT state, even in presence of frustration, is still a universal resource.

\section{CONCLUDING REMARKS}

We have shown that many 2D hybrid AKLT states (see Fig. 1) involving mixture of spin-2 and other lowerspin entities are also universal for MBQC. The results are nontrivial as they demonstrate that AKLT states with higher spins than $3 / 2$ can still be universal. We have also demonstrated that even though the spin-2 AKLT state on the kagome lattice (Fig. 1 d ) is not likely universal, one can decorate the lattice by adding additional sites so that the resultant AKLT state becomes universal. Moreover, the existence of geometric frustration does not necessarily destroy the universality, as demonstrated in spin-3/2 spin-1 hybrid AKLT state on the decorated star lattice. The following picture on the universality of AKLT states emerges: any 2D frustration-free lattice with any combination of spin-2, spin-3/2, spin-1 and spin-1/2, except that spin-2 sites are not neighbors, will host a universal AKLT state. To lift the constraint that the spin-2 sites are not neighbors, one would need to resolve the question question whether the spin-2 AKLT state on the square lattice remain universal. Furthermore, the effect of frustration may not be so severe as to completely destroy universality. Otherwise by simple decoration to remove frustration a resulting hybrid AKLT state can be universal. The family of $2 \mathrm{D}$ AKLT states contain many members that are universal, providing a nontrivial and versatile playground for quantum computation.

Acknowledgment. This work was supported by the National Science Foundation under Grants No. PHY 1314748 and No. PHY 1333903 (T.-C.W.) and by NSERC, Cifar and PIMS (R.R.).
[1] R. Raussendorf and H. J. Briegel, Phys. Rev. Lett. 86, 5188 (2001).

[2] F. Verstraete and J. I. Cirac, Phys. Rev. A 70 060302(R) (2004).

[3] D. Gross and J. Eisert, Phys. Rev. Lett. 98, 220503 (2007).

[4] H. J. Briegel, D. E. Browne, W. Dür, R. Raussendorf, and M. Van den Nest, Nature Phys. 5, 19 (2009).

[5] R. Raussendorf and T.-C. Wei, Annu. Rev. Condens. Matter Phys. 3, 239-261 (2012).

[6] M.A. Nielsen, Phys. Rev. Lett. 93, 040503 (2004). D.E. Browne and T. Rudolph, Phys. Rev. Lett. 95, 010501 (2005).

[7] K. Kieling, T. Rudolph, and J. Eisert, Phys. Rev. Lett. 99, 130501 (2007).

[8] R. Raussendorf, J. Harrington, and K. Goyal, Ann. Phys. (Amsterdam) 321, 2242 (2006).

[9] S. D. Bartlett, G. K. Brennen, A. Miyake, and J. M. Renes, Phys. Rev. Lett. 105, 110502 (2010).

[10] A. Miyake, Phys. Rev. Lett. 105, 040501 (2010).

[11] D. Gross, J. Eisert, N. Schuch, and D. Perez-Garcia, Phys. Rev. A 76, 052315 (2007).

[12] A. S. Darmawan, G. K. Brennen, and S. D. Bartlett, New J. Physics, 14, 013023 (2012).

[13] K. Fujii, Y. Nakata, M. Ohzeki, and M. Murao, Phys. Rev. Lett. 110, 120502 (2013).

[14] T.-C. Wei, Y. Li, and L. C. Kwek, Phys. Rev. A 89, 052315 (2014).

[15] D. V. Else, I. Schwarz, S. D. Bartlett, and A. C. Doherty, Phys. Rev. Lett. 108, 240505 (2012).

[16] J. Anders and D.E. Browne, Phys. Rev. Lett. 102,
050502 (2009).

[17] M. A. Nielsen, Rep. Math. Phys. 57, 147 (2006).

[18] X. Chen, B. Zeng, Z.-C. Gu, B. Yoshida, and I. L. Chuang, Phys. Rev. Lett. 102, 220501 (2009).

[19] J.-M. Cai, A. Miyake, W. Dür, and H. J. Briegel, Phys. Rev. A 82, 052309 (2010).

[20] See e.g. the review by L. C. Kwek, Z. Wei, and B. Zeng, Int. J. Mod. Phys. B 26, 1230002 (2012).

[21] M. Van den Nest, A. Miyake, W. Dür, and H. J. Briegel, Phys. Rev. Lett. 97, 150504 (2006).

[22] D. E. Browne, M. B. Elliott, S. T. Flammia, S. T. Merkel, A. Miyake, A. J. Short, New J. Physics 10, 023010 (2008).

[23] I. Affleck, T. Kennedy, E. H. Lieb, and H. Tasaki, Phys. Rev. Lett. 59, 799 (1987); Comm. Math. Phys. 115, 477 (1988). T. Kennedy, E. H. Lieb, and H. Tasaki, J. Stat. Phys. 53, 383 (1988).

[24] A. S. Darmawan and S. D. Bartlett , New J. Phys. 16, 073013 (2014).

[25] R. Ganesh, D. N. Sheng, Y.-J. Kim, and A. Paramekanti, Phys. Rev. B 83, 144414 (2011).

[26] A. Garcia-Saez, V. Murg, and T.-C. Wei, Phys. Rev. B 88, 245118 (2013).

[27] G. K. Brennen and A. Miyake, Phys. Rev. Lett. 101, 010502 (2008).

[28] T.-C. Wei, I. Affleck, and R. Raussendorf, Phys. Rev. Lett. 106, 070501 (2011).

[29] A. Miyake, Ann. Phys. (Leipzig) 326, 1656 (2011).

[30] T.-C. Wei, I. Affleck, and R. Raussendorf, Phys. Rev. A 86, 032328 (2012).

[31] T.-C. Wei, Phys. Rev. A 88, 062307 (2013). 
[32] Y. Li, D. E. Browne, L. C. Kwek, R. Raussendorf, and T.-C. Wei, Phys. Rev. Lett. 107, 060501 (2011).

[33] X. Chen, R. Duan, Z. Ji, and B. Zeng, Phys. Rev. Lett. 105, 020502 (2010).

[34] We thank an anonymous reviewer for pointing out that this can be regarded as an heralded error and the connection to percolation, similar to the consideration in the toric code by S. D. Barrett and T. M. Stace, Phys. Rev. Lett. 105, 200502 (2010).

[35] Whether it is an $X$ or $Y$ measurement depends on the POVM outcome of its neighbors, and can be straightforwardly deduced from the result in Ref. [30].

[36] M. Hein, W. Dürr, J. Eisert, R. Raussendorf, M. Van den Nest, and H.-J. Briegel, in Quantum Computers, Algorithms and Chaos, edited by G. Casati, D. Shepelyan- sky, P. Zoller, and G. Benenti, International School of Physics Enrico Fermi Vol. 162 (IOS Press, Amsterdam, 2006); also on arXiv:quant-ph/0602096

[37] One might want consider flipping from $F_{\alpha}$ to $K_{\alpha}$ uniformly with a probablity $p_{\text {flip }}=1 / 5$, consistent with the fact that the reduced density matrix of a single spin in the AKLT state is an idenity matrix (e.g. for spin-2 sites, it is $\mathbb{1} / 5)$ and that $\operatorname{Tr}\left(K_{\alpha}^{\dagger} K_{\alpha}\right) /\left[\operatorname{Tr}\left(F_{\alpha}^{\dagger} F_{\alpha}\right)+\operatorname{Tr}\left(K_{\alpha}^{\dagger} K_{\alpha}\right)\right]=$ $1 / 5$. However, it might be possible that the exact distribution differs very much from this independent and identical distribution (iid) such that the flipping with iid yields very different results from the exact distribution, even though we believe that this is not the case. The worst-case scenario provides the 'lower bound' on the universality. 
(a)

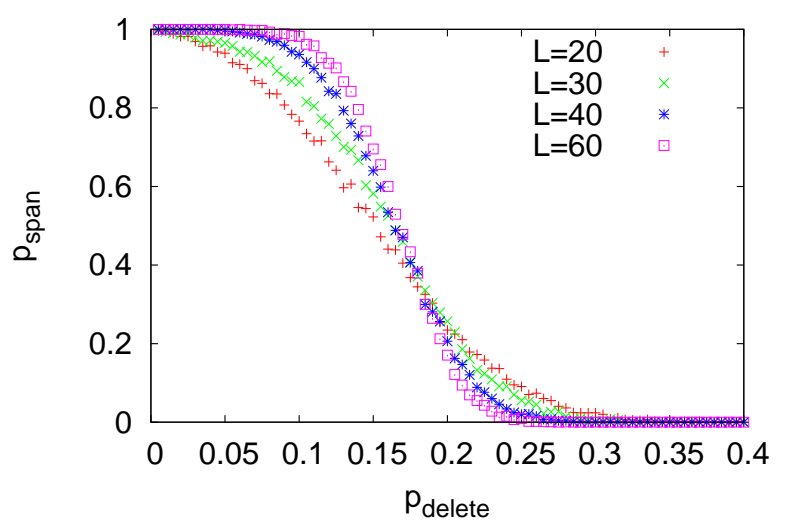

(b)

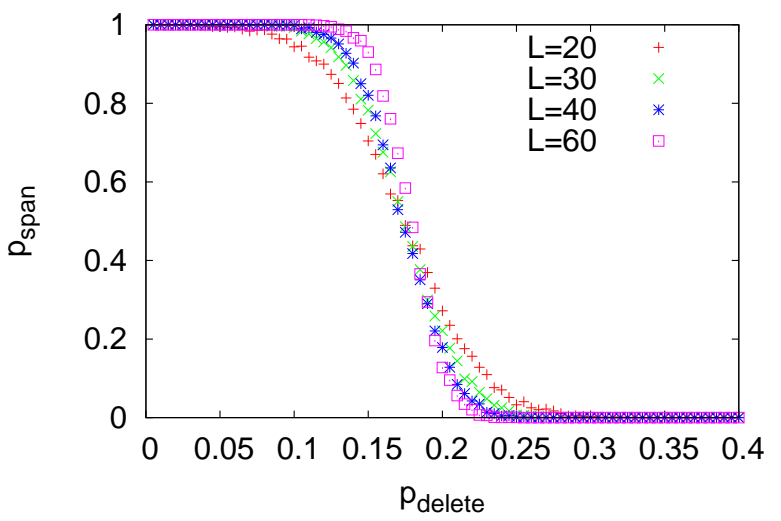

(c)

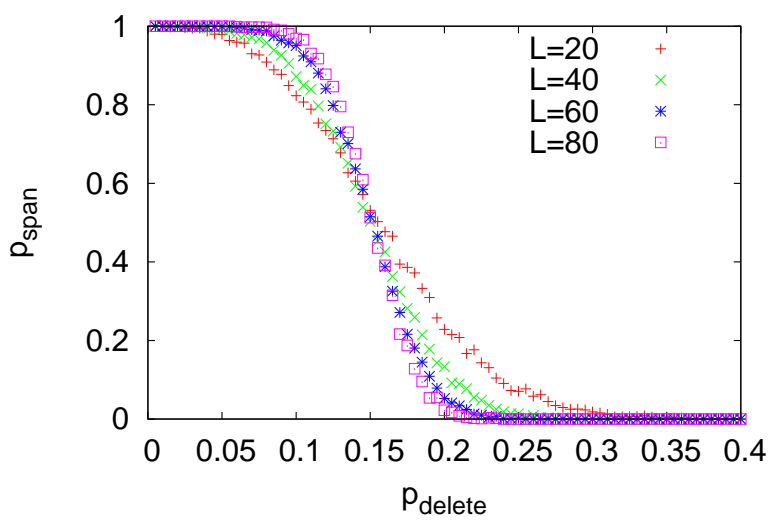

FIG. 4. (Color online) Site percolation study on the random graphs of domains resulting from the worst-case scenario procedure on the AKLT states on the lattices of (a) Fig. 17 with a total number of quantum spins (or sites) $N=5 L^{2}$, (b) Fig. 1 $1 \mathrm{~b}$ with $N=5 L^{2}$, and (c) Fig. 1 1 with $N=3 L^{2}$. Note that the quantities in both axes are dimensionless. In all cases, there is a phase transition (as measured by the probability of a spanning path $p_{\text {span }}$ ) from supercritical to subcritical phase as the probability of deletion $\left(p_{\text {delete }}\right)$ increases. The estimated thresholds of $p_{\text {delete }}$ are: $0.18(1), 0.19(1)$, and $0.11(1)$, respectively. Nonzero deletion thresholds show that the resulting random graphs after the procedure (before site deletion) are deep in the supercritical phase. 
(a)

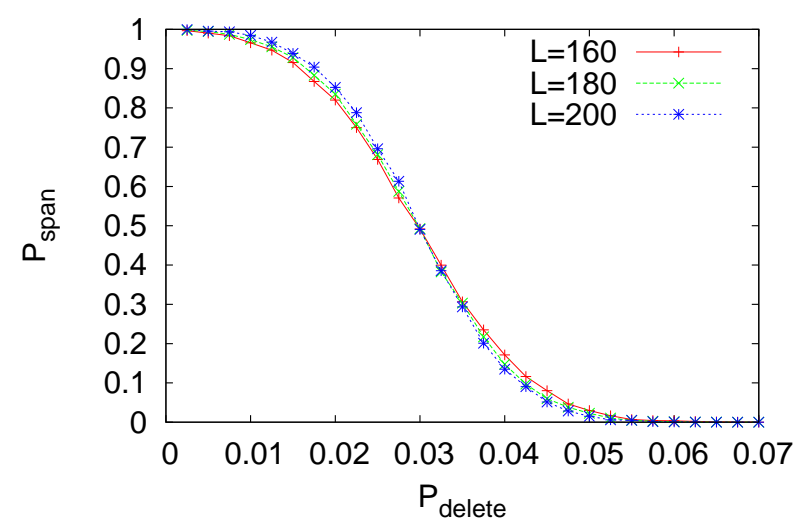

(b)

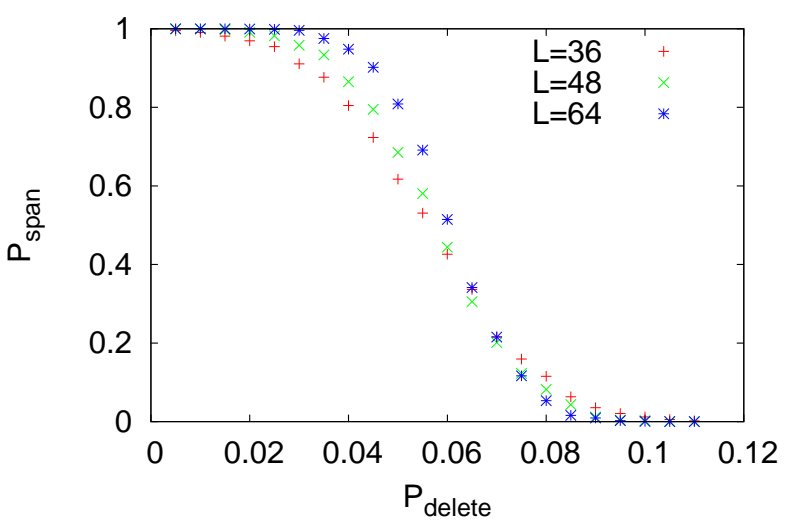

FIG. 5. (Color online) Site percolation study on the random graphs of domains resulting from the worst-case scenario procedure on the AKLT states on the lattices of (a) the decorated kagome, Fig. 1 1 , with a total number of quantum spins (or sites) $N=9 L^{2}$ and (b) the decorated star, Fig. 1f, with $N=9 L^{2}$. Note that the quantities in both axes are dimensionless. In both cases, there is a phase transition from supercritical to subcritical phase as the probability of deletion $\left(p_{\text {delete }}\right)$ increases (as seen from the crossing of $p_{\text {span }}$ curves for different sizes). In (a) the lines are used to guide the eyes. 